\title{
Designing a Playful Robot Application for Second Language Learning
}

\author{
Gijs Verhoeven ${ }^{1}$, Alejandro Catala ${ }^{2}$ and Mariët Theune ${ }^{1}$ \\ ${ }^{1}$ Human Media Interaction, University of Twente, The Netherlands \\ ${ }^{2}$ Centro Singular de Investigación en Tecnoloxías da Información (CiTIUS), University of \\ Santiago de Compostela, Spain \\ g.a.g.verhoevendstudent.utwente.nl, m.theunedutwente.nl, \\ alejandro.cataladusc.es
}

\begin{abstract}
Both storytelling and learning by teaching approaches have shown to be beneficial separately when integrating robots into learning settings. This paper discusses how to combine them, based on principles found in the literature, and presents the design and implementation of a playful robot application for second language (L2) learning targeted at primary school children. Our first tests showed that the robot kept the children engaged throughout the learning activity. It appears to be a promising approach as some vocabulary gain is observed in children, but there can be several factors involved that were not controlled in the trials and that will require further work.
\end{abstract}

Keywords: Interaction Design, Robot, Second Language Learning, Playful.

\section{Introduction}

In educational technology, robots are being introduced to facilitate learning and improve educational performance. Among the very different ways that robots can be integrated into learning settings, we are especially interested in exploring learning by teaching and storytelling. The former is a powerful approach as the stress is put on the children's activity, requesting them to perform some action that involves showing or explaining concepts to others. There is rich evidence for the effectiveness of this pedagogical approach, both in terms of learning outcomes and motivational effects [11]. In this approach, technology can be implemented as a teachable agent (TA), which plays the role of an entity that needs to be taught by the child. Initial research on learning by teaching with a TA was carried out with virtual TAs instead of robots. According to Chase et al. [5], students from 10 to 14 years old seemed to take responsibility for their (virtual) teachable agents and spent more time on their learning activities than when they learned by themselves. In recent years, there is growing interest in having a robot as TA $[6,7,11,13,15]$. Robots, being physical entities, might have a greater impact on children than virtual TAs [12]. This can help in the acceptance of the robot by the children and increase engagement and focus. More research is needed to explore potential advantages of a robot TA over a virtual TA.

Storytelling has been shown to be valuable in the development of children's skills such as linguistic skills, communication, logical thinking and creativity [8]. Robots 
have been used successfully to bring general exploratory storytelling activities (e.g. $[2,3,10])$. Concerning language learning, Kory and Breazeal [9] argue that children's language development is not just about exposure to words, it is also about embedding them in the context of a social interaction in which words are used to communicate meaning (a "dialogic context"). A storytelling activity can be a reason for such a dialogic context to arise. Besides this, they argue that robots can be beneficial as an intuitive physical interface for interaction, using easy interpretable social cues such as speech, movement and gaze. In addition, students can practice with a robot as often as they want [4].

In our work we combine learning by teaching and storytelling in a playful robot application for children's second language learning. Based on the previous points, this seems like a worthwhile approach, which to our knowledge has not been previously explored. The main question we address in this work is how to design a suitable language learning setting that combines a teachable robot and storytelling. Relevant issues to be addressed include how to integrate the content into a meaningful story, how to design the teachable agent in such a way that encourages children to teach the robot, and how to manage collaboration between children and the robot. This paper contributes to the design rationale of a robot application to support second-language learning (L2), and reports on preliminary tests carried out at school. The rest of the paper is organized as follows. Section 2 describes related work. Section 3 describes the design and development of the robot application. Section 4 reports preliminary user tests of the system, and Section 5 concludes by giving directions for future work.

\section{Related Work}

\subsection{Robots in Storytelling Activities}

Robots in storytelling activities for children have been used for diverse purposes. Kory and Breazeal [9] adapted the robot's language level to the children's level in a shared storytelling game and found that matching the robot's language skills improved native language learning outcomes for the child. Their research shows the benefit of a social and interactive environment, created via storytelling, for language learning. Giving the robot a slightly lower level possibly triggers learning by teaching.

The successful use of tangibles in a storytelling activity with a robot is shown in [10] and [14]. Krzywinski and Chen [10] designed a collaborative, tabletop storytelling activity with a robot as main character. Their small robot on wheels could be pushed and pulled by the children with tangible tiles, and successfully enhanced collaboration among students while telling a story together, passing tangibles and having discussions about the plot. However, due to technological constraints the robot was not advanced enough to fluently support the narrative. The children wanted the robot to express more behavior and emotions than it could. A possible solution to such technological constraints in research contexts is the use of a Wizard-of-Oz approach. This means that the robot is controlled by a person from a distance, which reduces the need for advanced and/or autonomous technology to allow a smooth 
interaction, as shown in [9]. The child is not aware of this, unless the effect of telling it is part of the experiment.

\subsection{Second Language Learning}

Some research has focused on second language learning (also called L2 learning) targeted at children. The effect of so-called word-word learning versus image-word learning has been investigated by Tonzar et al. [16]. Word-word learning means learning L2 words by being shown the first language (L1) translation. Image-word learning means learning L2 words by being shown an image of the meaning. Tonzar et al. conclude that image-word L2 language learning is more effective. It appears that this way, learned words stay longer in memory because they are coupled to the concept they express, instead of to the L1 word. They also confirmed that cognate words are easier to learn than non-cognate words. An L2 cognate word is a word which is similar to the L1 word in meaning and way of writing and/or pronunciation. For example, in English-French: letter and lettre or adorable and adorable.

A popular research topic in L2 learning for primary school children is the implementation of a social robot as tutor. Belpaeme et al. [1] provide an overview of such robots and present some guidelines for designing them. They argue that words should be taught in groups of the same subject (e.g. supermarket products or animals in the zoo) and within an appropriate context (e.g., an imaginary visit to the supermarket). In addition, they show the importance of repetition to learn an L2 word and its pronunciation. Finally, they state that introducing the robot as a peer could reduce anxiety and make sub-optimal interactions due to technical constraints more accepted by the child. Moreover, introducing the robot as a peer who needs to learn the language could lead to learning by teaching.

\subsection{Robot as a Teachable Agent in a Learning by Teaching Activity}

So far, there has not been much research on the effectiveness of robots used as teachable agents in learning by teaching. The main challenge is how to design the robot in such a way that it best helps learning for the student. Recent studies on robots as teachable agents in writing and reading activities have explored the effects of various design choices such as spatial placement and gesture use of the robot $[7,17]$. Jacq et al. [6] investigated how to achieve a "protégé effect", making the child feel responsible for the robot's learning. Keys to this were having an appealing scenario and the robot convincing the child of being a beginner who needed the child's help.

An important discussion among researchers in the field of teachable agents is whether a robot as TA is better than a virtual TA. Lindberg et al. investigate this in [11]. They conclude that both options work. The robot TA used in their experiment elicited more attention and enthusiasm amongst the students, compared to the virtual TA. However this enthusiasm decreased as the activity progressed. Also, compared to the virtual TA the robot was more limited in its behaviours due to technical and physical constraints. Increased enthusiasm for a robot compared to a virtual TA was also mentioned by Rosenthal et al. [13]. They did not find any differences between 
the different TAs in terms of perception of the system and linguistic alignment by children.

\section{Design Overview}

Below we discuss the design of our playful robot application for second language learning. As the basis for our design we used a so-called surfacebot, developed as an affordable social robot for use in schools [2]. The robot consists of two parts: a round base on wheels (about $20 \mathrm{~cm}$ diameter), and a tablet on top of it that forms the face and "brain" of the robot. We iteratively designed and tested a number of lofi prototypes before developing the final hifi prototype that was tested in a classroom experiment.

\subsection{Lo-Fi Paper Prototype}

From the works discussed in Section 2 we took several ideas that we incorporated in our design. To encourage learning by teaching, we framed the robot as a peer in need of help. To ensure that words are taught in coherent packages, we provided a context in the form of a storytelling activity with the robot as protagonist. We gave the robot emotions to increase the children's engagement with the robot. Since the use of tangibles is intuitive and appealing for children, we combined this with the imageword learning method. These ideas led us to a design in which the robot is an elephant who is visiting France, but does not know the language. Guided by the child, the elephant can visit different locations, represented by images on the tabletop (farm, beach, city, supermarket, mountains). At each location, the elephant finds three objects, represented by tangible tiles with images, which he can use in his adventures if he learns the French words for them (e.g., gold found in the mountains can be used to buy cheese in the supermarket).

We opted for a limited form of learning by teaching where the child does not need to have prior knowledge about the French words. Instead, the child sees the words displayed on a graphical interface, together with their corresponding image, and can "teach" them to the elephant by dragging them to an image of the elephant on the interface. When the elephant is taught a word he repeats it (in French) with a happy exclamation and a happy facial expression. After a while the elephant forgets the words (making him sad) to invite repeated teaching by the children, and thus repeated learning for the child.

To quickly check for pitfalls and potential design improvements, we created a lofi paper prototype of the application (see Fig. 1) and tested it with children in a local daycare centre. Our main target group are children aged between 7 and 8 . They are old enough to read and to make up a structured story, and young enough to still use toys in their stories. A first pilot test with one child (aged 9) revealed that we needed to provide more guidance and opportunities for storytelling. To this end, we changed some of the objects or put them in different locations. We also revised the sentences to be spoken by the elephant, so that they provided suggestions on what to do with the various objects. To set a clear end goal, the number of words to be taught to the elephant was restricted to eight. A second test with eight children (between 6-11 years 
old, average 8) showed that these revisions worked reasonably well, leaving the children with enough freedom to come up with their own story by deciding where to go and with what object to interact. We only needed to make some minor adjustments to the story-related sentences before finalizing the prototype. Because it was not fully clear to the children how they could interact with the tangible objects, in the hifi prototype we provided a way for the robot to physically carry a tile and move it around between locations.

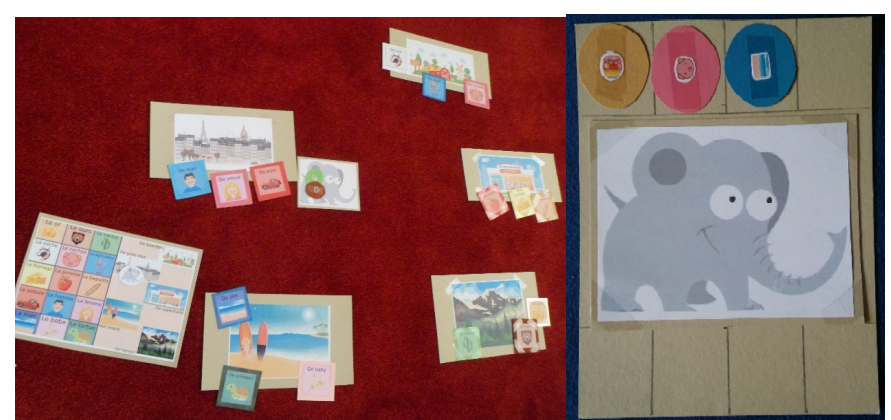

Fig. 1. Left) Lofi paper prototype; Right) Word-learning progression mock-up.

\subsection{Hi-Fi Prototype Implementation}

The hifi prototype implementation is based on three Android apps that are connected via wifi. One is for the surfacebot which implements the character (see Fig. 2-Left). It is able to show the elephant's emotional responses and play out the spoken utterances. Additionally, it provides a visual feedback reward by means of a colorful animation when a word is taught to the robot by children, and it displays the words that are learned. The robot has a mechanism to simulate the elephant forgetting a word and to request children to remind him, and reinforce learning this way. The word that is being forgotten is progressively being faded away on screen, and children should teach the word again to keep the elephant happy.

The second app is the Child UI, which is the panel controlled by the child (see Fig. 2-Right). On the left it has an array of tiles containing the word-image pairs in French, which can be dragged into the onscreen elephant on the right, in order to teach them to him and hear the word. The robot is intended to move autonomously within the physical playground, containing five different places. However, due to technical difficulties found in tracking the robot among the locations, we decided to remove this functionality for now. This required the child to move the robot physically between locations, but the core language learning activity could still be carried out.

Finally, we opted for tele-operation to prevent any adverse effects of technological constraints on the final evaluation. The Teleop app supports the activity manager (e.g. teacher or researcher) to operate and coordinate the responses of the robot following the Wizard-of-Oz approach. The utterances were pre-recorded in Dutch by an actor. They were specially designed to mediate interaction with the robot and support the storytelling and the learning by teaching strategy that was implemented. For example, there are sentences to ask the children to teach the robot (e.g. "X, can you teach me what that is in French?", where X is the teachable word in Dutch), to give feedback 
on the teaching and pronounce the French word (e.g. "Aha, so $\mathrm{X}$ is $\mathrm{Y}$ in French! Thank you!"), to request repetition (e.g. "X, what was that again in French? Could you teach me again?"), or to mediate and regulate the pace of the activity so that children discuss and make decisions on the story instead of just systematically trying to teach words (e.g. "Wait, I cannot learn words so fast").

The workflow of the activity is as follows. The robot introduces himself and his need to learn some French with the children's help on his way to the beach in France. Then children can explore going from one location to another, using the objects, and listening to the story suggestions given by the elephant. The objects can be used in the story by physically transporting the object tiles between locations using a tray fixed on the robot (see Fig. 2-Left). When children teach a word to the robot or fail to reteach on time, emotional responses and utterances are properly provided by the robot, controlled by the teleop in our implementation.

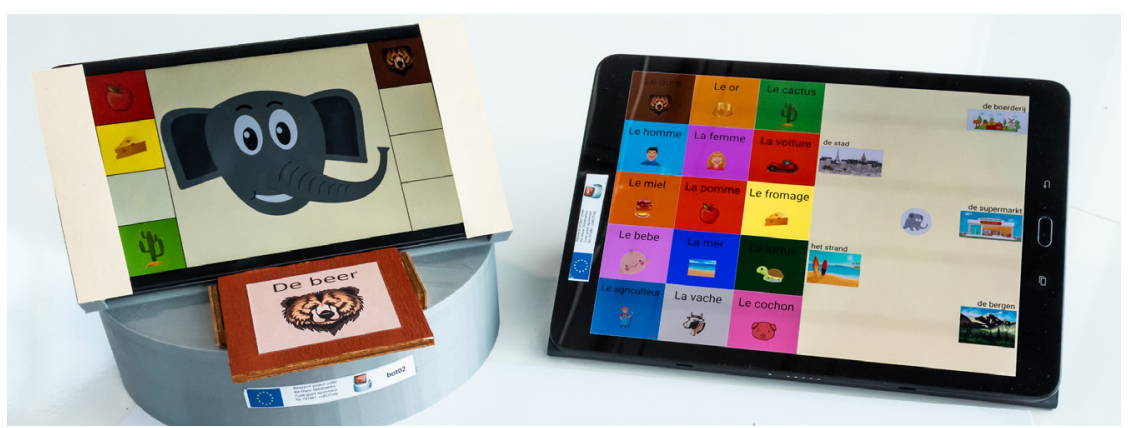

Fig. 2. Left) Elephant in a surfacebot; Right) Screen of the Child UI app.

\section{$4 \quad$ First Classroom Experiences}

We carried out a preliminary classroom experiment to test how the elements implemented in the prototype fit the purpose of second language learning. After getting approval from the Ethics Committee of our faculty and informed consent from the children's legal tutors/parents, 22 children with a mean age close to eight years old (13 male, 9 female) participated in an experimental playful activity at school with the developed prototype.

The method included a pre-test language questionnaire, participation in the activity with the prototype, and a post-test, carried out one day after. The language questionnaire was designed to easily gauge the children's pre-knowledge, filter initial guesses, and assess language gain by comparing the results between post- and pretests. It listed the French words that were available in the playful activity, showing the corresponding Dutch image-word pairs (in random order) on the sides (see Fig. 3Left). It included five French distractor words that were not considered in the activity in any way. To prevent issues such as spelling mistakes, children did not have to write but just connect words by drawing lines between them. They were clearly informed that the results would not count for their actual grades, and that they were not required to connect any words they did not know. The post-test questionnaire also included a few open questions to gather feedback on aspects of the activity the children liked and 
disliked. Pre- and post-test questionnaires were taken in the classroom for all children at the same time.

Children participated in the activity in pairs in a separate room. One child was the tablet operator, who was responsible for teaching the words to the robot. The other one was the robot operator, who had to move the robot to the right physical location on the tabletop and handle the tangible object tiles. Both roles remained unchanged during the activity. They had to cooperate, but their responsibilities and the way they experienced the materials were slightly different (see Fig. 3-Right). The activity lasted ten minutes. Video recordings were made during the activity to facilitate the review of any remarkable behavior. Children were informed after the post-test that teleoperation had taken place.

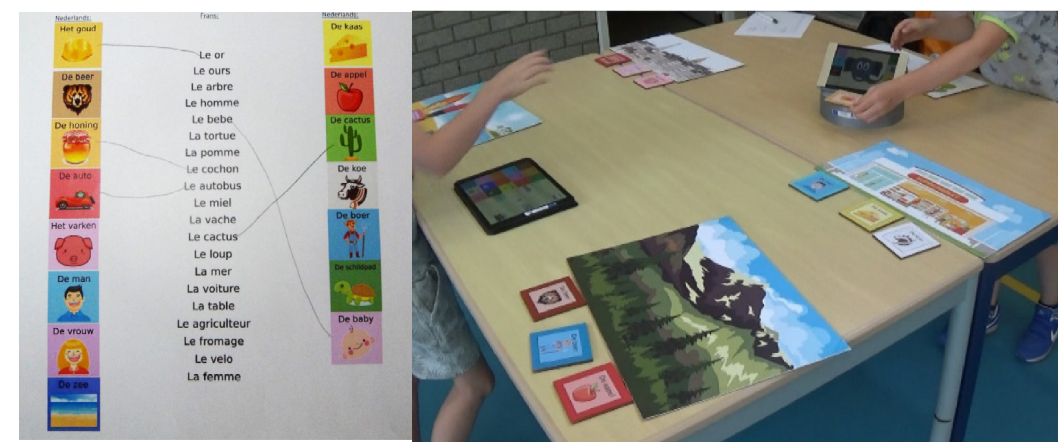

Fig. 3. Left) Language questionnaire. Right) groups playing during the learning activity, child putting the honey on the robot and discussing the action with the child behind the tablet.

The results of the language questionnaires showed that the children did not have any real knowledge of French prior to the activity. This was expected, as they did not have any formal education in that language yet. Even though the activity was short, it still had some positive effect in terms of language gain. On average, there was an overall gain of 2.05 words $(\mathrm{sd}=1.93$, mode $=$ median $=2, \min =0, \max =9)$ in the posttest compared to the pre-test. Fig. 4 shows the frequency of the words marked correctly by the children, split by their roles. Based on the findings about cognate words in the literature [16], it is very likely that this is the reason that le bebe and le cactus were learned so many times. This is probably also the reason that they were often already done correctly in the pretest.

We expected children operating the tablet, who had to teach the words to the robot, to have shown a higher gain than the robot operators, because of their more active role and increased exposure to the French words (which they could see on the tablet). However, both roles had similar gains $\left(\mathrm{m}_{\text {tablet }}=2.1, \mathrm{sd}=1.4\right.$, median $=2 ; \mathrm{m}_{\text {robot }}=2$, $\mathrm{sd}=1.09$, median=2). Firstly, we hypothesize that the learning activity was too short as to lead to differences in mean gain, as children in both roles remained highly engaged throughout the activity. This issue is something to be explored in a long-term intervention as discussed in the future work. We also noticed that the tablet operators had more words correct and exhibited a wider range of vocabulary in both the pre-test and the post-test. This may have been caused by the fact that children were allowed to 
choose freely which role they wanted to adopt. It is not unlikely that children with stronger language skills were more eager to adopt the tablet (active teaching) role.
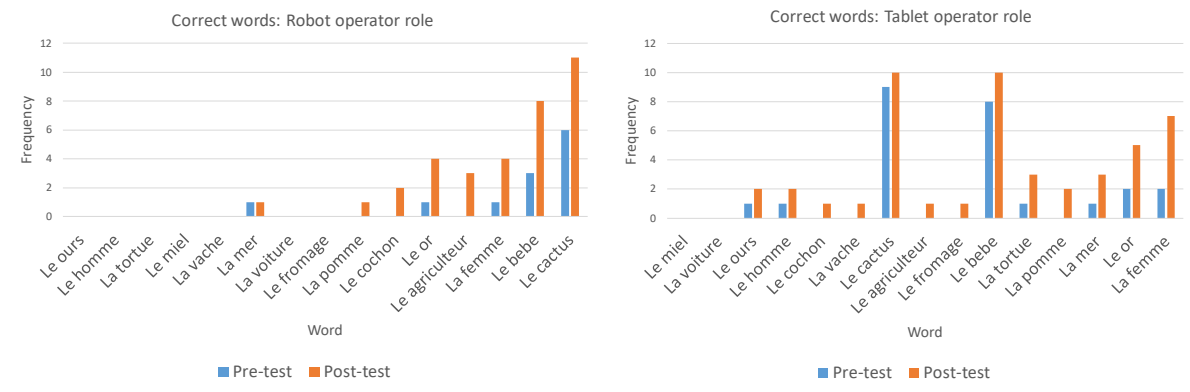

Fig. 4. Words correctly marked by children in the pre- and post-tests, by operator role: Left) robot operator; Right) tablet operator.

The overall learning gain displayed by both groups cannot be strictly and fully explained by the words taught to the robot, because not all of the gained words had been chosen by children to be taught. Possibly, some extra informal learning took place outside the learning activity. Afterwards, we overheard some children talking about the words, and the activity may also have triggered some additional discussion at home with parents, when the children explained what they did at school. Such extra informal learning cannot be reliably confirmed, but it is a plausible explanation. It would be in line with findings from Tanaka and Matsuzoe, who let children teach English words to a robot in the classroom. The children's parents reported that the children enjoyed the experience so much that they continued learning at home [15].

Regarding the questions to gather feedback, children showed positive responses overall. In many cases, they started with an unspecific everything (was liked). Typical specific responses for what they liked the most included 'learning new words', 'teaching the elephant new words', 'solving the tile puzzle', 'working with the robot', 'the French pronunciation by the robot', 'the elephant', and 'the robot'. It clearly shows that having a teachable robot was a focus of attention. Concerning dislike, just a few comments were made such as 'that you thought you had to control the elephant via the tablet', 'teaching the elephant again each time', 'dragging the circle to the elephant' and 'French'. Note that all these single more specific answers for both questions were given only once or twice.

In general, children seemed to be having fun while doing the activity. Smiles were often triggered when the robot started to talk "autonomously". Especially the French pronunciation of the robot made the children laugh or smile often. The children also said (some of the) French words themselves, either before or after teaching them to the robot. Communication between children often took place in the form of discussing where to go and what object the elephant should carry. Interaction with the system was performed similarly for all sessions; no single duo interpreted the activity significantly different from the others. Many different words were taught to the robot in different orders. There was no trend in which words were taught.

The clear affordance that the robot could carry an object, combined with the explanation that this was possible, changed the way the children did the activity, 
compared to the tests with the lofi prototype. Many children now perceived the activity as a puzzle where the objects needed to be moved to their correct location. Moving tiles to other locations was done constantly and was a major form of interaction with the application. The children perceived the robot's utterances as hints to where an object needed to go. This puzzle-like experience had a positive effect on the engagement with the activity. The re-teaching mechanic of the activity worked as expected. Many children noticed the sad face of the elephant and/or the fading away of a word before the elephant started to talk about this. They said things like "Quick, quick, quick, otherwise he will cry" or "He forgot something" or "He is crying!".

The children generally waited until the robot would say something, especially in the beginning. They let themselves be guided by the robot's hints. When the activity progressed, this waiting reduced and free play took over more. The children still listened to what the robot said, but interpreted this more freely.

\section{Conclusion \& Future Work}

We have developed a playful robot application for second language learning at primary schools. Our design relies on combining storytelling with the learning by teaching approach. It led us to consider spoken utterances as hints, which are part of the story. A strategy was implemented in the robot to forget words and request help from children to remember. The tests suggested there was some gain in words learned by children. However, it cannot be strictly and fully explained by the design of the activity, in particular with respect to the learning by teaching aspect, since not all the learning gains related to words that had been taught to the robot. A possible explanation is that some additional informal learning happened outside the classroom as a result of the activity at school. Also individual differences in children could have had an effect. For this reason, future work will focus on deploying a long-term intervention in such a way that we can explore the evolution of learning and robot interaction. Specific design aspects to be further explored are ways to strengthen the learning by teaching side of the application, e.g., by having this tie in with classroom activities giving the children prior exposure to the learning content to be taught to the robot and by giving the children a more active way of teaching, such as having to say more words or sentences themselves as part of a dialogue with the robot. It is also interesting to explore how learning can be supported more strongly by the puzzle behaviour that the children showed.

\section{Acknowledgement}

Research partially funded by H2020 MSCA-IF grant No. 701991 coBOTnity, European Regional Development Fund and Consellería de Cultura, Educación e Ordenación Universitaria (acc.2016-2019, ED431G/08). 


\section{References}

1. Belpaeme, T., Vogt, P., van den Berghe, R. et al.: Guidelines for designing social robots as second language tutors. International Journal of Social Robotics, 10(3), 325-341 (2018).

2. Catala, A., Theune, M., Gijlers, H., Heylen, D.: Storytelling as a creative activity in the classroom. In: Proceedings of the 2017 ACM SIGCHI Conference on Creativity and Cognition (C\&C '17), pp. 237-242, ACM, New York, NY, USA (2017).

3. Catala A., Theune M., Reidsma D., ter Stal S., Heylen D.: Exploring children's use of a remotely controlled surfacebot character for storytelling. In: Chisik Y., Holopainen J., Khaled R., Luis Silva J., Alexandra Silva P. (eds) Intelligent Technologies for Interactive Entertainment (INTETAIN 2017). LNCS, vol 215. Springer, Cham (2018)

4. Chang, C., Lee, J., Chao, P., Wang, C., Gwo-Dong, C.: Exploring the possibility of using humanoid robots as instructional tools for teaching a second language in primary school. Educational Technology \& Society, 13(2), 13-24 (2010).

5. Chase, C.C., Chin, D.B., Oppezzo, M.A., Schwartz, D.L.: Teachable agents and the protégé effect: Increasing the effort towards learning. Journal of Science Education and Technology, 18(4), 334-352 (2009).

6. Jacq, A., Lemaignan, S., Garcia, F., Dillenbourg, P., Paiva, A. Building successful long child-robot interactions in a learning context. 11th ACM/IEEE International Conference on Human-Robot Interaction (HRI), p. 239-246 (2016).

7. Johal, W., Jacq, A., Paiva, A., Dillenbourg, P.: Child-robot spatial arrangement in a learning by teaching activity. In: 25th International Symposium on Robot and Human Interactive Communication (RO-MAN'16), pp. 533-538. IEEE (2016).

8. Kocaman-Karoglu, A.: Telling stories digitally: an experiment with preschool children. Educational Media International, 52(4), 340-352 (2015).

9. Kory, J., Breazeal, C.: Storytelling with robots: Learning companions for preschool children's language development. In: The 23rd IEEE International Symposium on Robot and Human Interactive Communication, Edinburgh, 2014, pp. 643-648 (2014).

10. Krzywinski A., Chen, W.: Hi Robot: Evaluating Robotale. In: Proceedings of the 2015 ACM International Conference on Interactive Tabletops and Surfaces, ITS 2015, pp. 367$372,(2015)$.

11. Lindberg, M., Masson, K., Johansson, B., Gulz, A., Balkenius, C.: Does a robot tutee increase children's engagement in a learning-by-teaching situation? In: Intelligent Virtual Agents pp. 243-246 (2017).

12. Mubin, O., Stevens, C. J., Shadid, S., Al Mahmud, A., Dong, J. J.: A review of the applicability of robots in education. Technology for Education and Learning, 1, 1-7 (2013).

13. Rosenthal, A.M., Strassmann, C., Krämer, N.C.: Robots or agents - neither helps you more or less during second language acquisition. In: International Conference on Intelligent Virtual Agents, pp. 256-268, (2016).

14. Sylla, C., Coutinho, C., Branco, P.: A digital manipulative for embodied "stage-narrative" creation. Entertainment Computing, 5(4), 495-507 (2014).

15. Tanaka, F., Matsuzoe, S. Children teach a care-receiving robot to promote their learning: field experiments in a classroom for vocabulary learning. Journal of Human-Robot Interaction 1(1),78-95 (2012).

16. Tonzar, C., Lotto, L., Job, R.: L2 vocabulary acquisition in children: Effects of learning method and cognate status. Language Learning 59 (3), 623-646 (2009).

17. Yadollahi, E., Johal, W., Paiva, A., Dillenbourg, P.: When deictic gestures in a robot can harm child-robot collaboration. In: 17th Conference on Interaction Design and Children (IDC'18), pp. 195-206. ACM (2018). 